\title{
Studies on Affecting Factors of Wheel Slip and Odometry Error on the Performance of Wheeled Mobile Robots - A Review
}

\author{
D. Vidhya Prakash, A. Elango \\ Mechanical Engineering Department, Alagappa Chettiyar College of Engineering and Technology, India
}

\begin{tabular}{|c|c|}
\hline Article Info & ABSTRACT \\
\hline Article history: & $\begin{array}{l}\text { The wheeled mobile robots (WMR) find use for doing a variety of tasks in } \\
\text { different types of workspaces. These workspaces are form of ideal natural }\end{array}$ \\
\hline Received Nov 10, 2015 & flat, smooth terrain, unpredictable and irregular nature of these work spaces \\
\hline Revised Jan 20, 2016 & demanding a design challenges. Typically there work spaces may have pits, \\
\hline Accepted Feb 15, 2016 & $\begin{array}{l}\text { undulatory is not flat terrain, obstacles into tasks of the robot. There would } \\
\text { result in wheel slippage orodometry error. This task a toll in the form of }\end{array}$ \\
\hline Keyword: & $\begin{array}{l}\text { deviation from intended trajectory, navigation, travel time \&budgeted energy } \\
\text { consumption. These engineering issues can be addressed by proper design of }\end{array}$ \\
\hline Odometry error & wheel rotation, vibration amplitude of the robot body, travel time modulation \\
\hline Terrain & wheel slip \& odometry error is estimate of computational by design, pay load \\
\hline Trajectory & distribution, speed, wheel diameter, wheel width are the ply able design \\
\hline Wheel slippage & parameter in the designness scope. The effect of proper selection of the \\
\hline Wheeled Mobile Robot (WMR) & $\begin{array}{l}\text { values of there parameters is studied in this review. The paper reviews are } \\
\text { analyzing the basic\& direct effect of parameters are how to associate with } \\
\text { wheel slippage and odometry error and also which is the most affecting } \\
\text { factor on the wheeled mobile robots. }\end{array}$ \\
\hline
\end{tabular}

Copyright () 2016 Institute of Advanced Engineering and Science. All rights reserved.

Corresponding Author:

D. Vidhyaprakash,

Mechanical Engineering Department,

Alagappa Chettiyar College of Engineering and Technology

Karaikudi, Tamilnadu-630004, India.

E-mail: viddravid@gmail.com

\section{INTRODUCTION}

The robot is a electromechanical machine. The mobile robot is one of the type robots. The terms mobile isrobot, mobile platform, vehicle, robotare often. The wheeled mobile robot (WMR) is one of the type of mobile robot. The wheeled mobile robot has rolling wheels with a large mobility within its environment (air, land, and water), the total mobility relative to the sensing and reacting in the environment behavior is a perception ability of wheeled mobile robot. The wheeled mobile robots are using in various tasks\& workspace such as medical service, military surveillance, space exploration, forests, construction, material handling safety purpose in airports. In spite of the real time the WMR has been facing some inconvenient like a wheel slip, odometryerror, vibrations. This paper deals with wheel slip and odometry error. Robot swheelslip is relative motion between a wheel and terrain surface.

The slip is generally given as percentage of the difference between the surface speed of the wheel compared to the speed between axis and road surface as

Slip $(\omega r-v) / V$

Where

$\omega$ - Rotational speed of wheel 
$\mathrm{r}-$ Wheel radius

$\mathrm{v}-$ Vehicle Speed

The Most important tasks of mobile robots are to arrive at the target location. Wheeled mobile robots (WMR) with simple structure can move quickly on the flat and hard ground, however they cannot pass through the uneven or soft terrain. However in many practical applications such as navigation on outdoor or navigation on a slippery or uneven, irregular surface the no slip assumption are not satisfied. As a matter of fact, slip is required to generate traction force at the contact point that is responsible for the motion of the WMR. It is the possible to optimize the traction force and therefore the maneuverability of the WMR can be improved by controlling the magnitude of the wheel slip. It may generate instability in motion and this should be prevented. The slipping leads to large wastage of power\& time the actual wheel ground interaction needs to be considered in order to improve the robot movement. There is an increasing interest on mobile robots capable of traversing uneven terrain without slip. The wheel slip is leads to large wastage of energy of WMR is at a premium next part for applications such as planetary exploration.

And also the odometryerror is the use of data from motion sensor to estimate change in position over time. The odometry is used by some robots whether they be legged or wheeled to estimate their position related to a starting location. It also leads to localization errors and uncontrolled odometry. The odometry error is a measuring method of wheel rotation as a function of time. If the two wheels of robot are joined to a common axle or body relative to pervious orientation can be determined from theodometryerror measurement on both rear wheels of two wheeled mobile robot and single wheel is being in front of two wheeled mobile robots as a caster wheel to calculator. The variation in position and orientation of the robot with respect to starting point. The odometry error (oe) can be treated as output response and expressed as a function of parameters namely payload (L), speed (S), diameter of wheel (D) and thickness of wheel (T). To find out the variation in position and orientation of the robot with respect to starting point (p) across a given span of time ,liner distance DR and DL of each wheel traveled(computed from number of ticks of the encoders and diameter of the wheel)and wheel base(W)are substituted in the following equation the new orientation(R)in radians is calculated by

$$
\mathrm{R}=\mathrm{P}+(\mathrm{DR}-\mathrm{DL}) / \mathrm{W}
$$

Here the positioning in a wheeled mobile robot is an important task for many applications while navigation of the WMR. The positioning of wheeled mobile robots basically calculated using odometry from the wheel's encoder is mostly used for simple and inexpensive implementation for determine the relative localization of a mobile robot.

\section{LITERATURE-WHEELSLIP}

The tyre is slipping relative to the road the coefficient of friction for dynamic contact is lower, and have less traction. The slip is generally given as percentage of the difference between rotational speed (wheel speed) of the wheel and vehicle speed. The literature survey about the wheel slip of mobile robots is the mostly has been focused on the development of various models, stability analysis and path planning for mobile robots. But this review has consideration effect of parameters like payload, speed, geometry of wheels for mobile robot in the determination at wheel slip. Very few researchers have considered the effect of parameters like payload, velocity and geometry of wheels for mobile robot in the determination of relative localization using wheelslip \& odometryerror

\section{EFFECT OF PAYLOAD}

The weight carrying capacity is representing the payload it is not represent the separate weight of the wheeled mobile robot. In the based on concept the incremental range of payload of wheeled mobile robot is the considerably exist as a reduces the wheel slippage and tottering of robot wheel and structure. This result was found by T.Mathavaraj Ravikumar (1).

On the rigid terrain a large size wheel was suitable as it allowed a great amount of onboard battery mass, hence the increasing maximum give traversable ability.This result was found by Martin udenguard (4). While applying the particular range of load distribution apply on the wheeled mobile robot it will be controlling or reducing the wheel slippage. This result was found by T.MathavarajRavikumar (5). The maximum traction force required reducing as the maneuvers time increase. Which helps to reduce the maximum load on the actuator, this experiment was found by S. O. Oyadiji1 (7). While increasing the payload on the front wheel axle and improves traction and climbing abilities on all terrains and it will control

Studies on Affecting Factor of Wheel Slip and Odometry Error on the Performance ... (D. Vidhya Prakash) 
the wheelslip.this is determined by bruzzone (10). One of the prototype wheeled mobile robot weight distribution or the control of individual motors weight will sure improve the climbing ability of the robot, this was proved by Roland sieg wart (11), While the change the wheel load was applied, traction increased slightly with low inclination. The specific model "VENTRA" two wheeled mobile robot is being the useful utilization. The given figure is represent its detail and it self-weight is $1.2 \mathrm{~kg}$ and the distance between 2 wheels (W) is $120 \mathrm{~mm}$. the maximum speed is up to $200 \mathrm{~mm} / \mathrm{s}$.

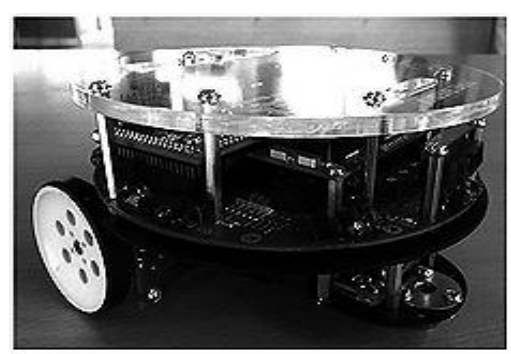

Figure 1. Souurce-International Journal of Scientific and Research Publications, Volume 3, Issue 12, December 20131 ISSN 2250-3153.

The robot was driven in an indoor environment as per the observation of experiment occur that the increasing the payload considerably reduce the wheel slippage and given smooth movement within the particular payload range $(0.8 \mathrm{~kg}-1 \mathrm{~kg})$ graph .

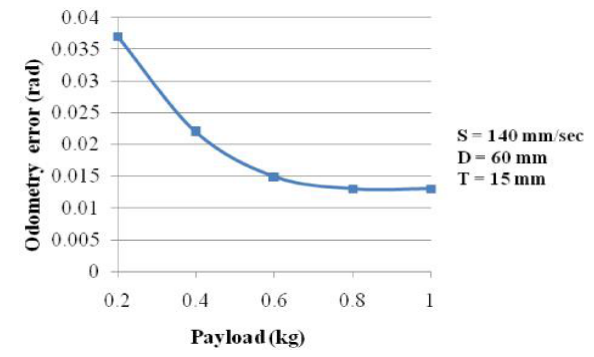

Figure 2. Source-International Journal of Scientific and Research Publications, Vol. 3, Issue 12, December 20131 ISSN 2250-3153.

The above graphical representation is producing or indicating the detail of wheel slip control ascending the load variance. This is proved by T.MathavarajRavikumar (13). The increasing in payload considerably reduces the wheel slippage and tottering of robot that leads to less deviation of WMR. The mobile robot rough terrain control control (RTC)for planetary exploration system commands increased torque to the rear wheel, which has a much higher load, this effect has been determined by karllagnemma (12). The split and fit trailing arm (SFTA) suspension mechanism system has control the load and leads to a $50 \%$ to60\% reducing the wheel slip of wheeled mobile robot. This effect has been proved by TharakeshwarAppala et al (16). Where ever changing the location of the load mainly affects the location of the global center of mass and the whole moment of inertia of the pendulum as presented before.this experiments has been determined by Khaled MK Goher et al (17).

\section{EFFECT OF SPEED}

Speed is related with velocity $(\mathrm{m} / \mathrm{sec})$ of mobile robot. Here we take the two wheeled differential drive mobile robot. When the wheeled mobile robot is moving the real-time practical conditions with slower speed graphical representation is given the useful range of speed $(140 \mathrm{~mm} / \mathrm{sec}-160 \mathrm{~mm} / \mathrm{sec})$.In this range of 
speed is cause the very minimal wheelslip \& wheel distortion less, his is founded by T. mathavaraja Ravikumar(1)

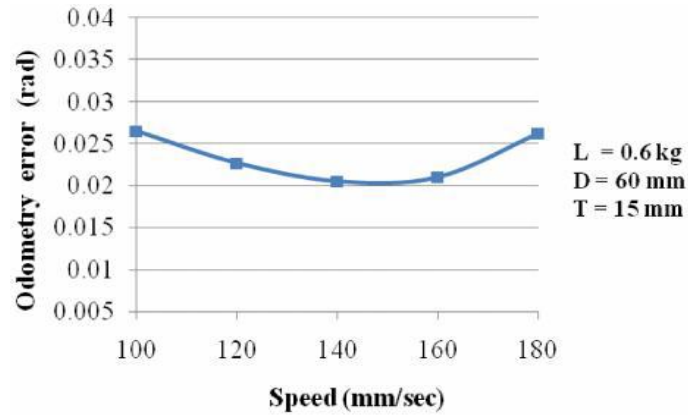

Figure 3. Source-ISSN2278-0149 www.ijmer, Vol.3, 1, January 2014.

When the particular range of velocity started to applying or increasing the wheel slip is also to increasing. This is founded by T.mathavaraja Ravikumar (5). The desired velocity profile is converted to a desired body frame velocity based on the robot's current position and orientation actual wheel velocity can be determined via odometry; however more sophisticated methods are required to estimate wheel slip. This result has been determined by Martin Udengaard (3). The system of sliding model control can actually improve the vibration phenomenon of mobile robots. It enables two-wheeled mobile robots control to achieve stable control function within short and limit time on the tracking control of position and speed. It can also promote the efficiency of system's control respond speed. This result has been produced byMao-Lin Chen (6). The specific model of wheeled mobile robot is determination the optimum condition for the better relative position. In this optimum level of parameter is represent the speed range is upto $144 \mathrm{~mm} / \mathrm{sec}$ is suggested to optimum condition for the better relative positioning of two wheeled differential drive robot, in this level wheel slippage is very low hence odometry is also better level,this effect has been studied byT.mathavaraj ravikumar(13).

When ever the robot can able to improve its successfully traverse the obstacle at low speed, the ballistic behavior must be allowed in high speed robot models as well as wheel slip and deformation. . This result has been produced by karllagnemma (18). Another view on the about that once slip ratio become larger than the boundary value of 0.2 , the control system will decrease the slip ratio value by reducing the rotational velocity of wheel, however, the traditional formula of slip ratio cannot be applied directly to the robot if the height is changing, because it is affected by the relative velocity between the body and wheel. This is founded by DINX XILUN (15). The steer angle is known, the wheel velocity along the forward (x) axis of the wheel frame can be determined from the $\mathrm{x}$ component the wheel equation in wheel coordinate then the drive velocity (around the axle) can be computed using the wheel radius.

Surface contact parameters were tuned to minimize wheel slip in the dynamic simulation. Minor disagreements between the physical experiment and simulation share due to unmolded peculiarities in Zo"e's construction, such as hysteresis in the roll-averaging mechanism. Has been produced by Alonzo Kelly (20). The overall effect is that the wheel velocities are matched more tightly even in the presence ofinternal and external disturbances. It should be also noted that the proposed control strategy does notrequire any additional sensors other than wheel encoders and steer potentiometers that are commonly available in most robots. ). This is founded by Giulio Reina (21).

\section{EFFECT OF DIAMETER OF WHEEL}

The wider wheel is minimizing ground pressure. A large size wheel has significantly less compaction deviation from linear path. The more advantage of large diameter wheel has more ground clearance and high. Centre of gravity and small diameter of wheel advantage has some merits they required less torque to traverse the same speed, with less weight. When the wheel slip is low as well as odometry error is also decreased while the diameterof wheel increased from $40 \mathrm{~mm}$ to $50 \mathrm{~mm}$ and increased when diameter increased further. It is clear that the odometry error is lesser between 50 to $60 \mathrm{~mm}$ of wheel diameter. The diameter of wheel should not be very high as well as very low for the reduction of odometry error. The lesser and larger diameters of wheel lead to the possibilities for wheel turn at maximum speeds and jerks at slower

Studies on Affecting Factor of Wheel Slip and Odometry Error on the Performance ... (D. Vidhya Prakash) 
speeds respectively which cause deviation from linear path, this effect has been studied by T. Mathavaraja Ravikumar (1).

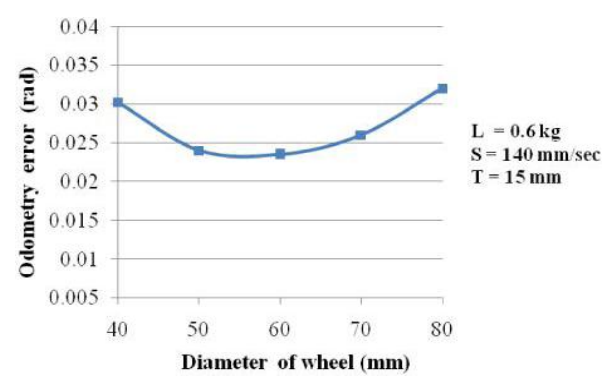

Figure 4. Source-ISSN2278-0149 www.ijmer, Vol, 3, 1, January 2014

The rear wheels of ROVER model robots are slightly large in diameter to keep proper contact with ground as and when front and middle wheels climb the step, smaller diameter wheels will provided low performance, due to closeness to ground casing links to interfere with the obstacle ,this effect has been studied by Debeshpradhan(2). The wider wheel is minimizing ground pressure. A large size wheel has significantly less compaction resistance than a small size wheel. A wider wheel was preferred for without slip or reduce the slip of wheel hence it for maximum traversable distance this effect has been studied by martin udenguard (4).

The moderate range of perimeter of robot's wheel diameter is occur the useful maneuverability of wheeled mobile robot, especially the very low and very high of wheel perimeter is not suitable for proper odometry and without wheelslippage. This effect has been studied by T. Mathavaraja Ravikumar (5). Based on confirmation of experiments for optimum condition of LOAD-1KG,SPEED-144MM/SEC,Dia OF WHEEL-60 mm, THICKNESS OF WHEEL-11mm of VENTRA two wheeled mobile robot has the optimum condition for the better relative positioning such as minimum odometry error the minimum odometryerror is come from low wheel slip of robot, this is proved by T. Mathavaraj Ravikumar (13). The active split offset caster (asoc) robot's position estimation based on traditional odometry methods may not be accurate in rough terrain. This is due to wheel slippage that causes miscounts of wheel rotation. In particular, the ASOC-driven omnidirectional mobile robot experiences large wheel slippage during sharp turning maneuvers. This is proved by GenyaIshigami (14).

\section{EFFECT OFTHICKNESS WHEEL}

The contact between Wheel and surface are decided the slippage, friction force. The thickness varied from $5 \mathrm{~mm}$ to $10 \mathrm{~mm}$ and further increased with the increase in thickness. So, it is evident that the minimum odometry error seems it come from lowest wheel slip in the range 10-15 mm

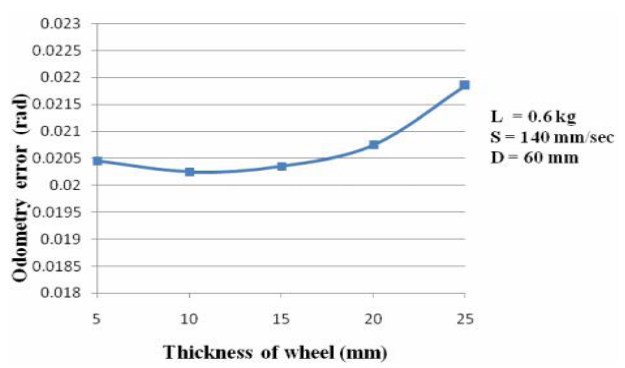

Figure 5. Source-ISSN2278-0149 www.ijmer, Vol, 3, 1, January 2014

of thickness. The lesser contact area of wheel on the floor due to smaller thickness provides the accurate wheel base for the odometry calculation that leads to minimal slippage and also low odometryerror. This is proved by T.mathavarajRavi Kumar (1). The rear wheels are slightly larger in diameter to keep proper 
contact with ground as and when front and middle wheels climb the step. This effect has been studied by Debeshpraan et al (2). Wheel-terrain interaction forces were determined via a simple coulomb friction model.The terrain elevation was modeled as a zero-mean triangular zed mesh with elevation points possessing a standard deviation of $\sigma$. In initial simulations it was assumed that the robot possessed perfect knowledge of terrain inclination.

Contact locations were determined by making a thin wheel approximation and finding the intersection are between the wheel and the local triangular mesh patches. This effect has been studied by Martin Udengaard and this is proved by Karl Iagnemma (3). The optimizations for the relatively deformable terrains (i.e., dry sand and snow) resulted in wheels with larger radii, but narrows width compared to those optimized for relatively rigid terrains. The large radii lead to decreased ground pressure and compaction resistance, while the thinner with lead to decreased wheel weight. This effect has been studied by Martin udengard (4). The lesser contact area of tyre on the floor due to smaller width provides the accurate wheel base for the odometry calculation that leads to minimal odometry error. This is founded by T. Mathavaraj Ravi Kumar (5).

Based on confirmation of experiments for optimum condition of LOAD-1KG, SPEED144MM/SEC, DIA OF WHEEL-60 mm, THICKNESS OF WHEEL-11mm of VENTRA two wheeled mobile robot has the optimum condition for the better relative positioning such as minimum odometry error the minimum odometry is come from low wheel slip of robot. This is founded by T.mathavarajRavi Kumar (13). Surface contact parameters were tuned to minimize wheel slip in the dynamic simulation. Minor disagreements between the physical experiment and simulations are due to unmolded peculiarities in Zoe's construction, such as hysteresis in the roll-averaging mechanism, this is founded by Alonzo Kelly (20).

\section{LITERATURE-ODOMETRY}

The odometryerror is another one of the factor to deciding to move in proper stream line, odometry is a measuring method of wheel rotations a function of time (real time). The wheel slippage is create the badodometry or odometryerror If the two wheels of the robot are joined to a common axle, or separate axle orientation of the center of single axle relative to the previous orientation. The odometryerror result (oe) can be treated as output response and expressed as a function of parameters (payload, speed, wheel dia, wheelwidth)

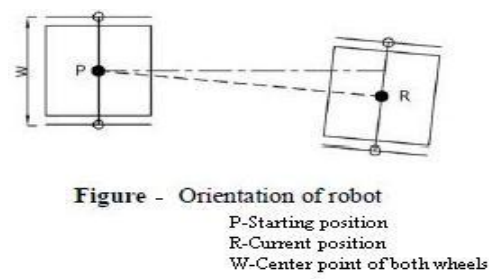

Figure 6. Source-ISSN2278-0149 www.ijmer, Vol, 3, 1, January 2014

\section{EFFECT OF PAYLOAD}

When the increasing of the payload the odometry error decrease. From the graphical representation the odometry error decreased with the increase of payload up to $0.8 \mathrm{~kg}$ also it was observed that there was no significant change in the odometry -error could be lesser between $0.8 \mathrm{~kg}$ and $1 \mathrm{~kg}$.

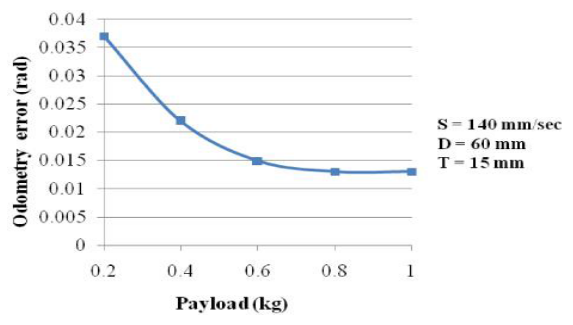

Figure 7. Source- ISSN2278-0149 www.ijmer, Vol, 3, 1, January 2014 
The increase in payload considerably reduce the wheel slippage and tottering of robot that leads to less odometry error. Has been produced by T. Mathavarajravikumaretal (1). In this case the RTC system commands increased torque to the rear wheel, which has a much higher load than the front wheel, resulting in increased net thrust. The dual criteria optimization remained in traction maximization mode for the majority of the travels. The moderate load is not allowed to wheelslip\&odometryerror. Has been produced by Karl Iagnemma (12). Wheel terrain contact locations were determined by making a thin wheel approximation and finding the intersection are between the wheel and the local triangular mesh patches. Has been produced byMartinUdengaard (3).

While applying the particular range of load distribution apply on the wheeled mobile robot is will be controlling or reducing the wheel slippage. Odometryerror, his is proved by T.Mathavaraj Ravikumar (5) The proposed robot has four wheels in order to maintain its stability when the center of gravity changes due to any extraload.Each wheel has attached to the tip of a leg because in many cases, sufficient space is not available to set the leg and wheel separately on the body of the robot. The proposed robot has four wheels in order to maintain its stability when the center of gravity the changes due to any extra load.This has been produced by d shuronakajima (9). The specific model "VENTRA" two wheeled mobile robot is being the useful utilization. The given figure is represent its detail and it self weight is $1.2 \mathrm{~kg}$ and the distance between 2 wheels (W) is $120 \mathrm{~mm}$, the maximum speed is up to $200 \mathrm{~mm} / \mathrm{sec}$. The robot was driven in an indoor environment as per the observation of experiment occur that the increasing the payload considerably reduce the wheel slippage and given smooth movement with in the particular payload range $(0.8 \mathrm{~kg}-1 \mathrm{~kg}) \mathrm{graph}$.

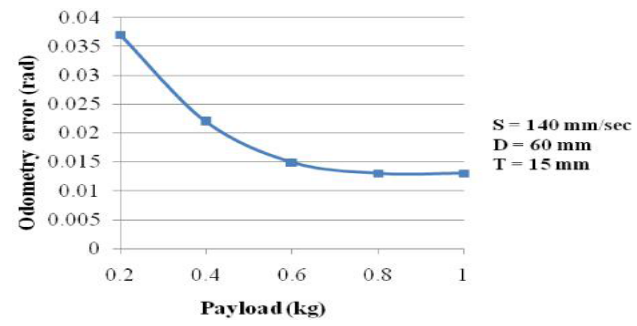

Figure 8. Source- ISSN2278-0149 www.ijmer, Vol, 3, 1, January 2014

The above graphical representation is producing or indicating the detail of odometry. Control accending the load variance. Has been produced by T. Mathavaraj Ravikumar (13). The increasing in payload considerably reduces the wheel slippage and odometry error, tottering of robot that leads to less deviation of WMR. When changing the location of the load mainly affects the location of the global center of mass and the whole moment of inertia of the pendulum as presented before. Has been produced by Khaled M. K Goher (17).

\section{EFFECT OF SPEED}

The speed factors are one of the important while speeds on the wheeled mobile robots that will be generate some disturbance, the effect of disturbance is allowed the speed range, when the robot is moving with slower speed in partial conditions, the possibilities of wheel distortions, vibrations and wheel slippage are appreciably less which causer very minimal odometry error, Has been produced by T. Mathavaraj Ravikumar (1).

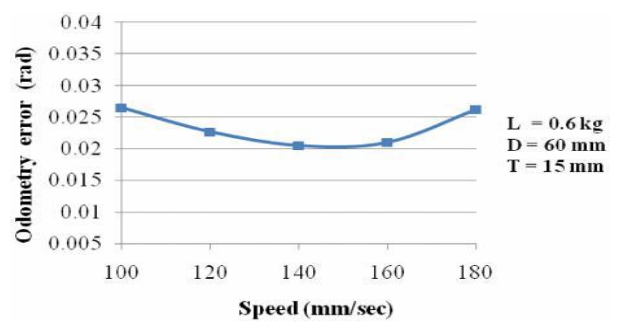

Figure 9. Source- ISSN2278-0149 www.ijmer, Vol, 3, 1, January 2014 
The desired velocity profile is converted to a desired body frame velocity based on the robot's current position and orientation. Actual wheel velocity can be determined via odometry; however moresophisticated methods are required to estimate odometryerror \& wheel slip by Martin Udengaard (3). from, the odometry error decreased significantly and started increasing after certain level. So, it is clearly understood that the error is minimum between $125 \mathrm{~mm} / \mathrm{secand} 150 \mathrm{~mm} / \mathrm{sec}$. When the robot is moving with slower velocity in practical conditions, the possibilities of wheel distortions, vibrations and wheel slippage are less which causes very minimal odometryerror.

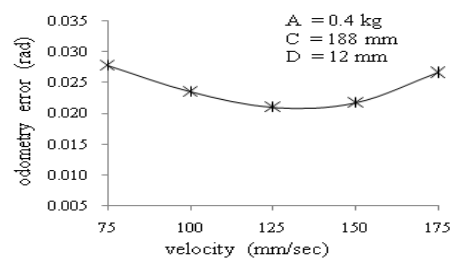

Figure10. Source- IJE TRANSACTIONS C; Aspects Vol. 27, No. 3 (March2014) 359-366

The diagram represent the normal level of the odometry $(125 \mathrm{~mm} / \mathrm{sec}-150 \mathrm{~mm} / \mathrm{sec})$ error is not increasing above has been produced by T. Mathavaraj Ravikumar (5). The system of sliding model control can actually improve the vibration phenomenon of mobile robots. It enables two-wheeled mobile robots control to achieve stable control function within short and limit time on the tracking control of position and speed. It can promote the efficiency of system's control respon speed. Has been produced by Mao-Lin Chen1 (6). Robot localization using the above odometric prediction (commonly referred to as dead reckoning) is accurate enough in the absence of wheel slippage and backlash. These effects are however largely reduced when the velocity is kept reasonably small and the number of backup maneuvers is limited. This is proved by Alessandro De Luca (8).

The specific model of wheeled mobile robot is determination the optimum condition for the better relative position. In this optimum level of parameter is represent the speed range is $144 \mathrm{~mm} / \mathrm{sec}$ is suggested to optimum condition for the better relative positioning of two wheeled differential drive robot, in this level odometry error is very low hence the motion is with in stream line. This is proved by T.mathavaraj ravikumar (13). The applied force or speed is activated on the particular area that reacting is the movement or deflection determined by Khaled M.K Goher (17). The wheel base area and speed of robots is decided the pose error of turning tracks. Determined by Changbae Jung (19). Large differences in the wheel velocities were produced with consequent, undesired increase in the amount of slippage the slip is producing the odometry error, the orientation error is defined as _ dr_dl/ where_dr and _ dl are the right and left side longitudinal displacement as measured by encoders Determined by Giulio Reina (21).

\section{EFFECT OF DIAMETER OF WHEEL}

From below graphical is represent the odometryerror decreased when the diameter of wheel increased from $40 \mathrm{~mm}$ to $50 \mathrm{~mm}$ and increased when diameter increased further. It is clear that the odometry error is lesser between $50 \mathrm{~mm}$ to $60 \mathrm{~mm}$ of wheel diameter. The diameter of wheel should not be very high as well as very low for the reduction of odometery error. The lessor and large distance (or) diameters of wheel lead to the possibilities for wheel turn at maximum speeds and jerks at slower speeds respectively which cause deviation from linear path. This is proved by T. Mathavaraj Ravikumar (1) .

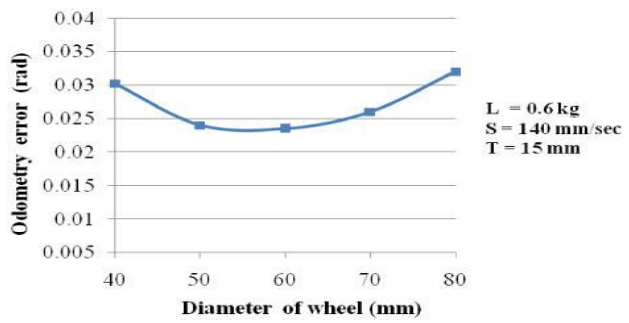

Figure11. Source-ISSN2278-0149 www.ijmer, Vol. 3, 1, January 2014 
The rear wheels of ROVER model robots are slightly large in diameter to keep proper contact with ground as and when front and middle wheels climb the step, smaller diameter wheels will provided low performance, due to closeness to ground casing links to interfere with the obstacle has been produced by Debeshpradhan (2). The odometry error decreased when the wheel perimeter increased from $126 \mathrm{~mm}$ to 157 $\mathrm{mm}$ and increased when perimeter increased further. It is clear that the odometry error is lesser between 157 to $188 \mathrm{~mm}$ of wheel perimeters. The wheel perimeter should not be very high not very low for the reduction of odometryerror. The lesser and larger perimeters of wheel lead to the possibilities for wheel turn at maximum speeds and jerks at slower speeds respectively which cause deviation from linear path.

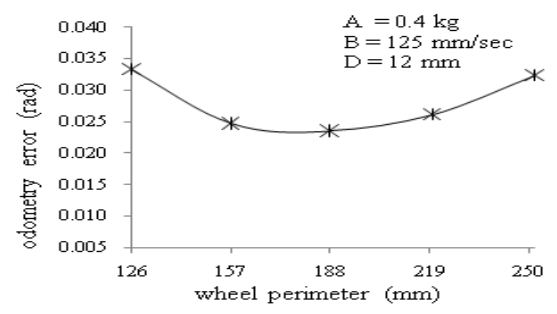

Figure 12. Source-IJE TRANSACTIONS C; Aspects Vol. 27, No. 3 (March2014) 359-366

Based on confirmation of experiments for optimum condition of LOAD-1KG, SPEED144MM/SEC, Dia OF WHEEL-60mm, THICKNESS OF WHEEL-11 mm of VENTRA two wheeled mobile robot has the optimum condition for the better relative positioning such as minimum odometry error the minimum odometry is come from low wheel slip of robot. Has been produced by T. Mathavaraj Ravi Kumar (13). The active split offset caster (asoc) robot's position estimation based on traditional odometry methods may not be accurate in rough terrain. This is due to wheel slippage that causes miscounts of wheel rotation. In particular, the ASOC-driven omnidirectional mobile robot experiences large wheel slippage during sharp turning maneuvers. Has been produced by GenyaIshigami (14).

\section{EFFECT OF WHEEL THICKNESS}

The contact between Wheel and surface are decided the slippage, friction force, thickness varied from $5 \mathrm{~mm}$ to $10 \mathrm{~mm}$ and further increased with the increase in thickness. So, it is evident that the minimum odometry error seems to be in the range $10-15 \mathrm{~mm}$ of thickness.

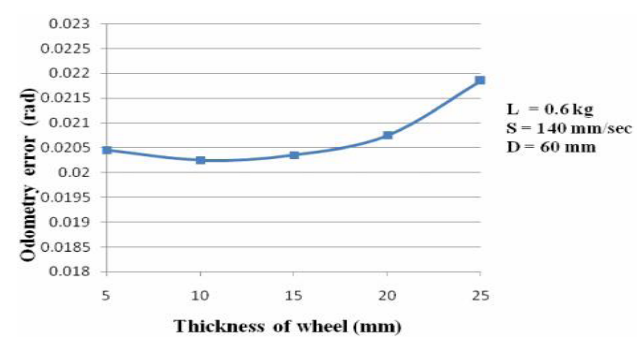

Figure 13. Source-ISSN2278-0149 www.ijmer, Vol. 3,1, January 2014

Lesser contact area of wheel on the floor due to smaller thickness provides the accurate wheel base for the odometry calculation that leads to lowodometry error. Has been produced by T. Mathavaraj Ravi Kumar (1). Wheel-terrain interaction forces were determined via a simple coulomb friction model. Terrain elevation was modeled as a zero-mean triangular zed mesh with elevation points possessing a standard deviation of $\sigma$. In initial simulations it was assumed that the robot possessed perfect knowledge of terrain inclination. Contact locations were determined by making a thin wheel approximation and finding the intersection are between the wheel and the local triangular mesh patches. This effect has been determined has been produced by Martin Udengaard and Karl Iagnemma (3). 
The optimizations for the relatively deformable terrains (i.e., dry sand and snow) resulted in wheels with larger radii, but narrows width compared to those optimized for relatively rigid terrains. The large radii lead to decreased ground pressure and compaction resistance, while the thinner with lead to decreased wheel weight. It will control the WMR, Has been produced, this effect has been determined by Martin udengard (4). There was a decrease in odometryerror when thetyre width varied from $4 \mathrm{~mm}$ to $8 \mathrm{~mm}$ and furtherincreased with the increase in width. So, it is evident that the minimum odometry error seems to be in the range $8-12 \mathrm{~mm}$ of thickness.

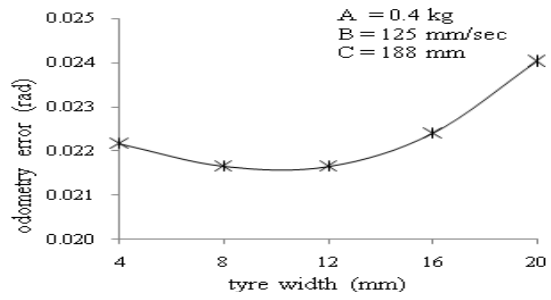

Figure 14. Source-IJE TRANSACTIONS C; Aspects, Vol. 27, No. 3 (March2014) 359-366

The lesser contact area oftyre on the floor due to smaller width provides the accurate wheel base for the odometry calculation that leads minimum error this effect has been determined by T.mathavaraj Ravi Kumar (5). Based on confirmation of experiments for optimum condition of LOAD-1KG, SPEED144MM/SEC, DIA OF WHEEL-60mm, THICKNESS OF WHEEL-11 mm of VENTRA two wheeled mobile robot has the optimum condition for the better relative positioning such as minimum odometry error the minimum odometry is come from low wheel slip of robot. This effect has been determined by T.mathavarajRavi Kumar (13). Surface contact parameters were tuned to minimize wheel slip in the dynamic simulation. Minor disagreements between the physical experiment and simulations are due to un modeled peculiarities in Zo"e's construction, such as hysteresis in the roll-averaging mechanism, Has been produced by Alonzo Kelly (20).

\section{COMPARSION WHEEL SLIP WITH ODOMETRY ON REAL TIME OF WMR (SUMMARY)}

The wheeled mobile robot's wheel slip and bad odometry are the mostly the affecting the robot's movement for differential two wheeled mobile robot. The both draw backs (slip. Odometry error) are creating some another indirect effect parameters namely Jerk moving, vibrations, pitching, yawing, rolling etc. Here the analysis first about the wheel slippage of wheeled mobile robot how to be reach the target with in the time being and energy saving condition. In case wheeled mobile robot's both wheels are affecting in wheel slippage it will be not give the any navigation, any one wheel is affecting in the wheelslip it navigation is slowdown on any terrain or floor. The odometry error is the another one of the affecting factor the wheel slippage is creating the bad odometry. It is the different from the wheelslip because the movement are there toward the target, but the odometryerror is producing the uncontrolled stream tracking movement. The travelling time, energy loss is more. From the above analysis the time consuming and energy loss are mostly wastage by the wheelslip and odometry error time of navigation. But the most facing factor in the real time of navigation on WMR to achieving the target/goal, because the mobile robot's movement may not possible while the affecting both wheels of WMR. Any one wheel affecting it will occur the slow movement. In the odometry error time the movement is possibilities, hence the target reaching is possibility in even or uneven condition.so it is too help to reach the target/goal, comparing with the wheel slip factor.

\section{CONCLUSIONS}

From the above studies the followings are the conclusions:

The wheel slip \&odometryerror of wheeled mobile robots is mostly affecting factors\&direct affecting factors to the robots performance.Especially the wheel slip lead to more time consuming and more energy wastage of WMR comparing with odometryerror.The direct affect parameters (Payload, speed, diameter of wheel, thickness of wheel) and very influencing on the WMR performance to producing the wheelslip\&odometryerror.When affecting the most of all wheels by the wheel slippage on the any surface/floor it's performance will be reduce suddenly, its recovery may be difficulty (sea sand surface,lose 
soil).The bad odometry, jerk, vibration,pitching\&yawing are possible to producing/ rising in the time of wheel slip,. Hence the target reaching is not reliable.To this end the four main parameters (load, speed, diameter of wheel, thickness of wheel) of WMR have been considerable with reference to the selection or recommend range of parameters to make a innovative in order to the mobility performance (without slip, good odometry) is most significant condition but also overall system of WMR is reliability. Whenever the frictionalforce acting on the apparent contact area in order to stop the slipping or burningout, motion of wheel.

Future trend in mobile robotics are also briefly discussed that I can be said that two wheels must having individual axle and motor. If the front wheel is much weight compare than both rear wheels of wheeled mobile robot, it will be give and maintain its linear motion control by the formula $\mathrm{F}=\mathrm{ma}$,(f-force, $\mathrm{m}$ mass, a-accelaration).andthe frictional force acting on the apparent contact area in order to stop the slipping or burningout, motion of wheel. Also rear wheels are rolling and keep in proper stream line. It may be a better than previous for high end application in outdoor units robots (military and floor utility tasks) especially low cost wheeled mobile robot.

\section{REFERENCES:}

[1] T.Mathavaraj Ravikumar., R. Saravanan., "Optimization of relative position in a two wheeled differential drive robot", ISSN2278-0149 www.ijmer, vol. 3, No. 1, January 2014.

[2] Debesh Pradhan, jishnu Sen-Desigh and development of an automated all-terrain wheeled robot, Advances in RoboticsResearch,vol,1,No,1(2014)021-039.

[3] Martin Udengaard., Karl lagnemma., "Kenematic Analysis and Control of an Omnidirectional Mobile Robot in Robot Terrain, 77Massachusetts Ave," Massachusetts Institute of Technology, Cambridge, MA02139.

[4] Martin Udengaard., Karl lagnemma., "Design of an omnidirectional Mobile Robot for Rough Terrain", Massachusetts Institute of Technology, Cambridge, MA 02139 USA.

[5] T.Mathavaraj Ravikumar., R.Saravanan., "Reduction of odometry Error in a Two Wheeled Differential Drive Robot”, IJE TRANSACTIONS C, Aspects, Vol. 27, No. 3 (March2014) 359-366.

[6] MAO-linChen.,YANG-Hsiung., "Slider Controller Design for Two Wheeled Mobile Robot Scheme", 100, 11J OURNAL OF CCIT, Vol. 40, No. 2, NOV, 2011.

[7] S. O. Oyadiji., A.Ayalew., "Analytical framework for the smooth manoeuvre of wheeled mobile robots traversing obstacles”,Proc,R,Soc,A(2005) 461,481,507,doi;10,1098/rspa.2004,1337.

[8] Alessanodro De Luca, Giuseppe Oriolo, "Control of Wheeled Mobile Robots; An Experimental Overview", Dipartimento di Informatica e systemistica, Universithdegli Studi di Roma "la sapienza”, Italy.

[9] Shuro Nakajima., "Development of Four-wheel-type Mobile Robot for Rough Terrain and verification of its Fundamental Capability of Moving on Rough Terrain", 2-17 Tsudanuma, Narashino, chiba 275-0016, JAPAN.

[10] L. Bruzzone., G. Quaglia., "Review article; locomotion systems for ground mobile robots in unstructured environments", Mech science, 3, 49-62, 2012, doi; 10.5194/ms-3-49-2012.

[11] Roland Siegwat., Pierre Lamon., "Innovation design for wheeled locomotion in rough terrain", Robotic and Autonomous System 40 (2002)151-162.

[12] Karl Lagnemma., Steven Dubowsky., "Mobile Robot Rough Terrain control (RTC) For Planetary Exploration “, Massachusetts institute of Technology Department of Mechanical Engg Cambridge, MA 02139 USA.

[13] T. Mathavaraj Ravikumar., R. Saravanan., "Modeling and Optimization of Odometry Error in a Two Wheeled Differential Drive Robot", International Journal of Scientific and Research Publications, Vol. 3, Issue 12, December 2013, ISSN 2250-3153.

[14] Genya Ishigami., Elvin Pineda., "Performance Analysis and Odometry Improvement of an Omnidirectional Mobile Robot Robot for Outdoor Terrain", 2011IEEE/RSJ International Conference on Intelligent Robot and System, September 25-30, 2011, San Francisco,CA,USA.

[15] Ding Xilum., LI Kejia., "Dynamics and wheel's slip Ratio of a wheel-legged Robot in wheeled Motion considering the change Height”, Doi;103901/CJME201205, Chines Journal of Mechanical Engineering, April 10, 2012.

[16] Tharakeshwar Appala., Ashitava Ghosal., "A Three-Wheeled Mobile Robot for Traverse Uneven Terrain Without Slip", Simulation and Experiments.

[17] Khaled MK Goher., Mo Tokhi., "Modelling, Simulation and Balance Control of a Two Wheeled Robotic Machine with Static Variation in Load Position", ISBN; 98-0-9553018-5-8/ISBN; 978-0-9553018-6-5 (CD).

[18] Karl Lagnemma., Dariusz Golda., "Experimental study of High-speed Rough-terrain Mobile Robot Models for Reactive Behaviors", Cambridge MA02139 USA.,

[19] Chang bae Jung., Woojin Chung., "Calibration of kinematic parameters for Two Wheel Differential Mobile Robots by Using Experimental Heading Errors", International journals of Advanced Robotic Systems, 2011 jung and Chung, License Intech.

[20] Alonzo Kelly., Heal Seegmiller., "A Vector Algebra Formation of Robot Velocity Kinematic”, Robotic Institute, Carnegie Mellon University.

[21] Giulio Reina., "Cross Coupled Control for All Terrain Rovers”, Sensors 2013,13,785-800;doi.3390/s130100785. 